\title{
Development of a biophysical screening model for gestational hypertensive diseases
}

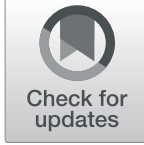

\author{
Sharona Vonck ${ }^{1,2^{*}}$ D, Anneleen S. Staelens ${ }^{2}$, Dorien Lanssens ${ }^{1,2}$, Kathleen Tomsin², Jolien Oben², \\ Liesbeth Bruckers ${ }^{4}$ and Wilfried Gyselaers ${ }^{1,3}$
}

\begin{abstract}
Background: To investigate the possibility of using maternal biophysical parameters only in screening for the different types of gestational hypertensive diseases.

Methods: A total of 969 pregnant women were randomly screened in first and second trimester, of which 8 developed Early-onset Preeclampsia, 29 Late-onset Preeclampsia, 35 Gestational Hypertension and 897 women had a normal outcome. An observational maternal hemodynamics assessment was done via standardized electrocardiogram-Doppler ultrasonography, Impedance Cardiography and bio-impedance, acquiring functional information on heart, arteries, veins and body fluid. Preliminary prediction models were developed to test the screening potential for early preeclampsia, late preeclampsia and gestational hypertension using a Partial Least Square Discriminant Analysis.
\end{abstract}

Results: A combined model using maternal characteristics with cardiovascular parameters in first and second trimester offers high screening performance with Area Under the Curve of 99,9\% for Early-onset Preeclampsia, 95,3\% for Late-onset Preeclampsia and 94\% for Gestational Hypertension.

Conclusions: Using biophysical parameters as fundament for a new prediction model, without the need of biochemical parameters, seems feasible. However, validation in a large prospective study will reveal its true potential.

Keywords: Gestational hypertensive diseases, Hypertension, Preeclampsia, Screening, Biophysical parameters, Prediction

\section{Background}

Gestational hypertensive disorders (GHD), including gestational hypertension (GH), preeclampsia and essential hypertension, contribute to maternal and perinatal mortality and morbidity, and are linked to health problems in later life [1]. Their aetiology is considered multifactorial, is still not completely understood and troubles prediction. Aside chronic hypertension, onset of GHD's develop in the second half of pregnancy. However, a detection of subclinical signs in the first half of pregnancy opens perspectives for prophylactic treatment (low dose aspirin) or preventive measures (blood pressure recording) towards reduction of the adverse maternal and neonatal outcome [2].
Current screening programs are based on a combination of maternal characteristics (age, ethnicity, body mass index, nulliparity, family or personal history) with biophysical (mean arterial pressure, pulsatility index) and biochemical markers (soluble Fms-like tyrosine kinase-1 (sFlt-1), placental growth factor (PlGF), pregnancy-associated plasma protein A (PAPP-A)), offering reported detection rates of $>90 \%$ for early-onset preeclampsia (EPE) at 10\% false positive ratio. However, for late-onset preeclampsia (LPE) or $\mathrm{GH}$, those models are troubled with a rather poor performance (LPE: 35-76\%; GH: 18-21\%) [3-5]. Other efforts were done seeking other biomarkers [6], metabolites [7], aorta intima media thickness [1], flow mediated dilatation of the brachial artery [8] or proteomics [9], but neither of them were highly promising.

\footnotetext{
* Correspondence: sharonavonck@hotmail.com

${ }^{1}$ Faculty of Medicine and Life Sciences, Hasselt University, Agoralaan, 3590

Diepenbeek, Belgium

${ }^{2}$ Department of Obstetrics \& Gynaecology, Ziekenhuis Oost-Limburg,

Schiepse Bos 6, 3600 Genk, Belgium

Full list of author information is available at the end of the article
}

(c) The Author(s). 2019 Open Access This article is distributed under the terms of the Creative Commons Attribution 4.0 International License (http://creativecommons.org/licenses/by/4.0/), which permits unrestricted use, distribution, and reproduction in any medium, provided you give appropriate credit to the original author(s) and the source, provide a link to the Creative Commons license, and indicate if changes were made. The Creative Commons Public Domain Dedication waiver (http://creativecommons.org/publicdomain/zero/1.0/) applies to the data made available in this article, unless otherwise stated. 
In hypertensive disorders, it is already shown that cardiovascular parameters differ from the first trimester onwards and that different sets of parameters relate to different types of hypertension [10, 11]. We aimed to investigate the strength of using these maternal "type-specific" biophysical parameters only as screening to predict the different types of gestational hypertensive diseases.

\section{Methods}

\section{Definitions of gestational hypertensive disorders}

Diagnoses were made during the course of the normal clinical care by the investigators according to the ISSHP guidelines [2] and based upon the patient's clinical record. After birth, gestational outcome was defined and data were categorized in normotensive pregnancy (NP), GH, EPE, LPE, essential hypertension (EH) and all classified according to birth weight percentile $\leq$ or $>10 \%$ as small for gestational age (SGA) or non-SGA respectively. $\mathrm{GH}$ was defined as a non-proteinuric hypertension, developed after 20 weeks of gestation. Preeclampsia was defined as new-onset hypertension with proteinuria $\geq 300 \mathrm{mg} / 24 \mathrm{~h}$, labelled as early-onset at clinical presentation of $<34$ weeks and late-onset at presentation of $\geq 34$ weeks of gestation. EH was a non-proteinuric hypertension with need for antihypertensive medication, developed < 20 weeks. GH, LPE and EPE were used for analysis (Appendix).

\section{Study design}

Approval of the Ethical Committee was obtained before study onset (MEC ZOL, reference: 13/090 U) and informed consent before inclusion. One group of inclusions were normotensive women with singleton pregnancies, between $8^{+0}-15^{+6}$ weeks of gestation, presenting at the obstetric ultrasound scanning clinic at Ziekenhuis Oost-Limburg Genk for their routine first trimester ultrasound scan who were invited to participate in an observational study between 2011 and 2016 where a cardiovascular assessment was performed. The second group were women with established risk factors, such as concommittant disease, obstetric or medical history, referred by their gynaecologist to our maternal-fetal medicine unit. The cardiovascular assessment was repeated between $18^{+0}-27^{+6}$ weeks of gestation in $64 \%$ of the patients. The other $36 \%$ of patients were lost to follow-up for logistical reasons or delivered elsewhere. At delivery, all included pregnancies were categorized according to confirmed and documented outcome. For this preliminary analysis, we included patients with NP, GH and LPE diagnoses, all without SGA neonates to obtain unbiased groups with a pure hemodynamic profile (Appendix). As exception, the group of EPE patients were included with and without SGA neonates, partly from the theoretical concept that poor foetal growth is an intrinsic feature of EPE and partly to increase the numbers of pathological cases as they were otherwise too low (Appendix). Pregnancies complicated with EH (with or without SGA, $n=28$ ), superimposed late preeclampsia with or without SGA $(n=5)$, HELLP with or without SGA $(n=3)$ and isolated SGA $(n=92)$ were excluded from the prediction model analysis, as well as multiplet pregnancies $(n=2)$ (Appendix).

\section{Maternal characteristics}

Maternal characteristics were obtained through history taking and retrospective review of the medical records for each patient. This included maternal age, pregestational weight, height, pregestational body mass index (BMI), allergy, parity, history on diabetes/intra-uterine death/intra-uterine growth restriction/thrombophilia/ hypertension.

\section{Cardiovascular profile}

A maternal cardiovascular profile was taken in every pregnant woman combining three non-invasive techniques (Impedance cardiography, ECG-Doppler ultrasound and bio-impedance) to obtain functional information about the complete maternal circulation (arteries, veins, heart, and body fluid content) (Table 1). A standardized proto$\mathrm{col}$, as described below, was used as reported in previous other studies [12-14].

\section{Impedance Cardiography (ICG)}

The Non-Invasive Continuous Cardiac Output Monitor (NICCOMO, Medis Medizinische Messtechnik GmbH, Ilmenau, Germany) was used for standardized automated sphygmomanometric blood pressure measurement on the right arm and with an appropriate cuff width. Impedance cardiography was performed using four electrodes (two on the axillary line under the thorax and two in the neck) eliminating skin resistance. The examination was performed after stabilization of cardiovascular function in standing position. Parameters were classified as blood pressures [diastolic (DBP), mean arterial pressure (MAP)], flow parameters [heart rate $(\mathrm{HR})$, stroke volume (SV), cardiac output $(\mathrm{CO})]$, contractility parameters [pre-ejection period (PEP), left ventricular ejection time (LVET), velocity index (VI), acceleration index (ACI)] and vascular parameters [total peripheral resistance (TPR)]. The latter was calculated using the formula $(\mathrm{MAP} \times 80) / \mathrm{CO}[15,16]$.

\section{ECG-Doppler ultrasound}

An electrocardiogram was combined with Doppler ultrasonography of the maternal renal interlobar veins, hepatic veins and the arcuate uterine arteries 
Table 1 Overview of all parameters derived in one cardiovascular assessment session with the three techniques

\begin{tabular}{llll}
\hline & ECG-Doppler & ICG & Bio-impedance \\
\hline Heart & & Heart Rate (HR) \\
& Stroke Volume (SV) & Cardiac Output (CO) \\
& & Pre-ejection Period (PEP) \\
& Left Ventricular Ejection Time (LVET) \\
& & Velocity Index (VI) \\
Arteries & Acceleration Index (ACI) & Total Peripheral Resistance (TPR) \\
& Left + Right Arterial Pulse Transit (APT) & Diastolic Blood Pressure (DBP) & \\
& Left + Right Resistivity Index (RI) & Mean Arterial Pressure (MAP) & \\
& & & \\
Veins & Hepatic Venous Pulse Transit (VPT) & & \\
& Left and Right Renal VPT & & Total Body Water (TBW) \\
& Hepatic Vein Index (HVI) & & Extracellular Water (ECW) \\
Renal Interlobal Vein Index (RIVI) & & \\
Fluid & & &
\end{tabular}

ECG electrocardiogram, ICG impedance cardiography

using a $3.5 \mathrm{MHz}$ transabdominal probe during interrupted breathing in supine position (Aplio $\mathrm{Mx}$, Toshiba Medical Systems nv, Sint-Stevens-Woluwe, Belgium). Each parameter was measured three consecutive times and averaged, reducing intra-variability [17]. Parameters of arteries and veins were classified into 2 groups: pulse transits and impedance indices.

The heart rate corrected venous pulse transit (VPT) is the time interval between the P-top from the ECG-wave and the A-wave from the Doppler pulse wave (PA in $\mathrm{ms}$ ). In the arteries (heart rate corrected arterial pulse transit, APT), the time interval starts at the Q-wave on the ECG and ends at the start of the Doppler end-diastolic point D (QD in ms). The pulse transits are adjusted for heart rate, which is variable due to advancing gestation, and thereby divided by $\mathrm{RR}$ (time interval in ms between two consecutive R-waves of the ECG signal) [18].

At the venous side, the maximum and minimum flow velocity is measured from the renal and hepatic Doppler signal. An impedance index is calculated using the formula [(Maximum Velocity-Minimum Velocity)/Maximum velocity] [13, 19]. This renal interlobar vein index (RIVI) and hepatic vein index (HVI) are considered the venous equivalents of the arterial Resistivity Index (RI) which is calculated by the formula (Peak systolic velocity - End diastolic velocity)/Peak systolic velocity. In the uterine arcuate arteries, RI and Pulsatility Index (PI, (Peak systolic velocity - minimal diastolic velocity)/Mean velocity) were measured as reported [20, 21].

A combination of TPR and APT in dyn.sec/cm², was also calculated and describes the relation between vascular resistance in the systemic vs. the uterine circulation (uterine flow promoting peripheral resistance (UFPPR)).

\section{Bio-impedance}

The body composition and fluid balance were measured by a multiple frequency bioelectrical impedance analyzer (Maltron Bioscan 920-II, Maltron International LTD, Essex, UK) in supine position with stretched arms and legs, without socks or shoes [22]. Two electrodes, receiving the electrical signal, were placed on the dorsal surfaces of the wrist and ankle at the level of the process of the radial and ulnar resp. fibular and tibial bones. Two other electrodes, sending the electrical signal, were attached to the third metacarpal bone of the right hand and right foot. The applied current was $0,6 \mathrm{~mA}$ with a frequency of $5,50,100$ and $200 \mathrm{kHz}$ during $5 \mathrm{~s}$. Total Body Water (TBW) was estimated, as the total of intracellular water $(\mathrm{ICW})$ and extracellular water $(\mathrm{ECW})$.

\section{Statistics}

An independent $\mathrm{t}$-test at 5\% significance level was used for intergroup comparison of continuous demographic data. Chi-square test was used for categorical demographic variables. Normality was checked via Shapiro-Wilk. Data were presented as mean \pm SD or $n(\%)$. These analyses were done in SPSS (SPSS Inc., Chicago, Illinois, USA).

The preliminary prediction models were calculated using a Partial Least Square Discriminant Analysis (PLS-DA), which discriminates NP from GH, NP from EPE and NP from LPE. All measured biophysical parameters were converted first to multiples of the expected normal median (MoM) per trimester. The PLS-DA evaluated the performance using (A) demographic data only; (B) demographic data + the maternal biophysical 
parameters at 12 weeks and (C) demographic data + the maternal biophysical parameters at 12 and 20 weeks. All pregnancies were used in the training set and cross-validation was used to quantify the performance. The number of observations in the training set decreased substantially when using multiple sets of biophysical parameters (ultrasound, impedance cardiography and bio-impedance variables) and data from both trimesters, due to missing data in one of the sets. Sensitivities, specificities, positive predictive values, negative predictive values, receiver operating curves (ROC) and likelihood ratios presented in this manuscript, were obtained with the PLS-DA with cross validation. SAS (SAS 9.4, Institute Inc., Cary, NC USA) was used for this data analysis.

\section{Results}

A total of 969 pregnant women were measured at their first ultrasound appointment. Eight were categorized as EPE, 29 LPE, 35 GH and 897 NP. Patient and outcome characteristics of the four outcome groups are shown in Table 2.

The best performance was obtained by combining the maternal biophysical parameters of first and second trimester together with the maternal characteristics. More specifically this means, the models include a set of demographical variables: maternal age, allergy, parity, length, BMI, history of hypertension/thrombophilia/ IUGR/intra-uterine death/diabetes, and three sets of biophysical parameters (presented in Table 1), measured in first and second trimester (Fig. 1). Positive likelihood ratios (LR) are between 7,27-7,40 and the negative between 0 and 0,18 .

\section{Early preeclampsia}

The detection rate (DR) of EPE is $100 \%$ with false positive ratio (FPR) of $0 \%$. Positive predictive value (PPV) is $67 \%$ and negative predictive value (NPV) is $100 \%$. The area under the curve (AUC) is 99,8\% (Fig. 1a).

\section{Late preeclampsia}

At $14 \%$ FPR, the detection rate is $100 \%$. The PPV is $21 \%$ and NPV $100 \%$. The AUC for LPE is $94 \%$ (Fig. 1b).

\section{Gestational hypertension}

The GH model is characterized by a detection rate of $84 \%$ at $12 \%$ FPR. This provides a PPV of $23 \%$ and NPV of $99 \%$. The AUC is $95,3 \%$ (Fig. 1c).

\section{Discussion}

This study is the first to demonstrate the feasibility of screening for all types of GHD using maternal biophysical parameters in combination with demographic parameters. Even without inclusion of biochemical parameters, our prediction models already show good performances (AUC of $>94 \%$, DR of $>84 \%$ ), but it is expected that these will improve further by combining these biophysical parameters with biochemical parameters. Validation in a large prospective study is now needed to evaluate the potential and applicability of biophysical parameters in screening for GHD.

Our study is the first to assess all major components of the maternal circulation as one integrated functional circle: volumes, heart, arterial and venous hemodynamics and are used as fundaments in a preliminary prediction model. A standardized protocol of non-invasive techniques with reported inter- and intra-observer correlations is used [12-14]. The bio-impedance technique may be criticized as being less valid than maternal echocardiography or dye dilution plasma volume measurements, however our results are in line with these so- called gold standard methods [23] and with other reports [24]. It should be appreciated that, similar to other reported methods [25], the bio- impedance methodology is very easy to perform with very low inter- and intra-observer variabilities, allowing a general application with a minimum of training or expertise. The total cardiovascular protocol is cheap and time efficient $(15 \mathrm{~min})$. We acknowledge that all pathological cases were rather low in this study and that EPE was based on very few measurements, even with inclusion of SGA and non-SGA, which might bias the model of EPE. The statistics behind the models is also still too complex and preliminary to provide us already with a defined algorithm with corresponding coefficients. Another point of attention is that 13.5\% of our studied population has diabetes mellitus and uses insulin for therapy with potential interference with our cardiovascular measurements.

Current guidelines on screening and management of women at high risk for hypertension in pregnancy are based on maternal risk factors only, and performance is rather low with $35 \%$ detection rate for preeclampsia and $40 \%$ for early preeclampsia [26, 27]. In practice, diagnosing GHD is based on elevated blood pressures and/or proteinuria during a routine clinical visit in late second or third trimester, and at that stage, disease is already established and preventive therapy is not useful anymore. Therefore, various models were investigated to improve first trimester prediction. The current best approach combines maternal characteristics with first trimester uterine artery Doppler pulsatility index, MAP and a combination of biomarkers (PIGF, sFlt-1 or PAPP-A), reaching detection rates of $>90 \%$ for EPE and between 35 and $76 \%$ for LPE by a $10 \%$ FPR [3-5]. This means 
Table 2 Patient and outcome characteristics in the four outcome groups

\begin{tabular}{|c|c|c|c|c|}
\hline & $\begin{array}{l}\text { Normotensive Pregnancy } \\
\text { (NP) }(n=897)\end{array}$ & $\begin{array}{l}\text { Early-onset Preeclampsia } \\
(\mathrm{EPE})(n=8)\end{array}$ & $\begin{array}{l}\text { Late-onset Preeclampsia } \\
(\mathrm{LPE})(n=29)\end{array}$ & $\begin{array}{l}\text { Gestational Hypertension } \\
(\mathrm{GH})(n=35)\end{array}$ \\
\hline \multicolumn{5}{|l|}{ Characteristics at inclusion } \\
\hline Maternal age, years & $30(27-33)$ & $30(25-36)$ & $30(27-34)$ & $30(27-33)$ \\
\hline Pregestational maternal weight, kg & $64(58-74)$ & $76(64-84)^{*}$ & $66(62-82)$ & $65(59-79)$ \\
\hline Pregestational maternal height, $\mathrm{cm}$ & $166(161-170)$ & $166(163-170)$ & $166(165-170)$ & $167(162-170)$ \\
\hline Pregestational $\mathrm{BMI}, \mathrm{kg} / \mathrm{m}^{2}$ & $23,3(21-26,8)$ & $27,3(23,3-29,8)$ & $24,1(22,4-30,2)$ & $23,6(22-27,9)$ \\
\hline \multicolumn{5}{|l|}{ Gestational age at assessment, weeks+days } \\
\hline First trimester & $12 w 2 d(11 w 5 d-12 w 5 d)$ & $13 w(12 w 3 d-13 w 2 d)^{*}$ & $11 w 5 d(11 w 1 d-12 w 5 d)^{*}$ & $12 w 2 d(11 w 6 d-12 w 5 d)$ \\
\hline Second trimester & $20 w 2 d(19 w 6 d-20 w 5 d)$ & $21 w(20 w 3 d-26 w 3 d) *$ & $20 w 5 d(20 w 1 d-21 w 4 d)^{*}$ & $20 w 2 d(19 w 5 d-20 w 6 d)$ \\
\hline \multicolumn{5}{|l|}{ Medical history } \\
\hline Diabetes Mellitus & $116(12,9 \%)$ & $2(25 \%)$ & $6(20,7 \%)$ & $6(17,1 \%)$ \\
\hline Thrombophilia & $33(3,7 \%)$ & $1(12,5 \%)$ & $1(3,4 \%)$ & $2(5,7 \%)$ \\
\hline Allergy & $177(19,7 \%)$ & $0(0 \%)$ & $4(13,8 \%)$ & $13(37,1 \%)^{*}$ \\
\hline \multicolumn{5}{|l|}{ Obstetric history } \\
\hline Intra-Uterine Death & $15(1,7 \%)$ & $0(0 \%)$ & $1(3,4 \%)$ & $1(2,9 \%)$ \\
\hline Intra-Uterine Growth Restriction & $7(0,8 \%)$ & $0(0 \%)$ & $0(0 \%)$ & $1(2,9 \%)$ \\
\hline Nulliparity & $472(52,6 \%)$ & $4(50 \%)$ & $8(27,6 \%)^{*}$ & $14(40 \%)$ \\
\hline \multicolumn{5}{|l|}{ Hypertension } \\
\hline Family history & $41(4,6 \%)$ & $1(12,5 \%)$ & $1(3,4 \%)$ & $2(5,7 \%)$ \\
\hline Previous pregnancy & $55(6,1 \%)$ & $2(25 \%)$ & $4(13,8 \%)$ & $10(28,6 \%)^{*}$ \\
\hline \multicolumn{5}{|l|}{ Outcome characteristics } \\
\hline Birth weight, g & $3425(3145-3734)$ & $1300(572-1712)^{*}$ & $3230(2690-3656)^{*}$ & 3405 (3040-3720) \\
\hline Birth weight, percentile & $57,5(33-77,5)$ & $7,5(3,8-48,8)$ & $63,8(28,1-79,4)$ & $52,5(23,5-82,5)$ \\
\hline Gestational age at delivery, weeks + days & $39 w 5 d(38 w 5 d-40 w 3 d)$ & $31 w 5 d(27 w-32 w 5 d)^{*}$ & $37 w 5 d(36 w 5 d-39 w 6 d)^{*}$ & $39 w 3 d(38 w 5 d-40 w 5 d)$ \\
\hline
\end{tabular}

Data are presented as median (IQR) or $n$ (\%)

${ }^{*} p<0,05$ was considered significant different from normotensive pregnancy (NP)

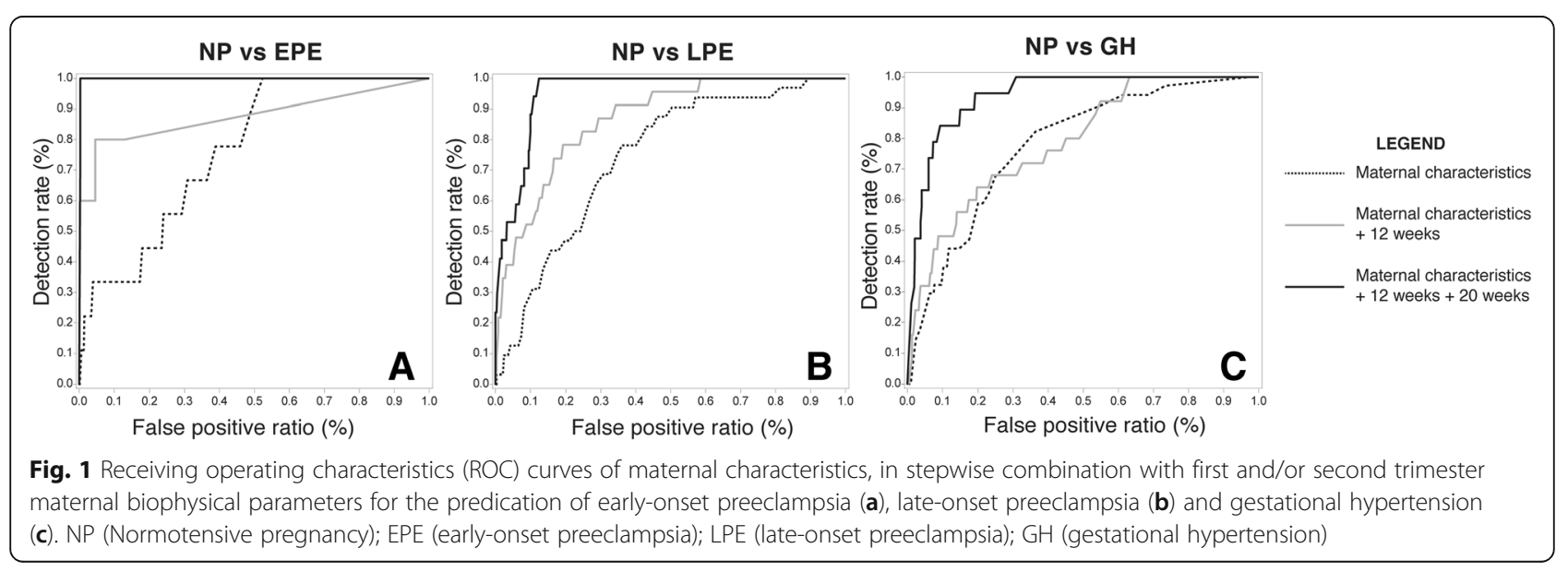


EPE has a good detection rate, but performance on LPE is poor $[3-5,26]$. Prediction of GH scores even lower $(18-21 \%$ at $5 \%$ FPR) [5]. Compared to those latter models, the prediction models in our study promise better (preliminary) detection rates on all diagnoses.

In multiple studies [10], an extended list of pathologic biophysical parameters are measured already in the first trimester: higher uterine pulsatility indices, higher TPR, lower $\mathrm{ACI}$ and VI, higher $\mathrm{CO}$ and $\mathrm{SV}$, etc. We have shown before that type-specific cardiovascular characteristics allow discrimination between GH, EPE and LPE $[11,21]$. More and more studies use maternal hemodynamics to explain the pathophysiology of preeclampsia and other hypertensive disorders. It is important to focus not only on (early) preeclampsia, but on all types of hypertension when creating a prediction tool. Therefore, we aimed to explore a different fundament for a prediction model than the current popular research tracks, and focus on all hypertensive cases.

The models in our study showed the best performance when adding the first and second trimester parameters together. Other prediction models use only first trimester information. However, it is shown that all women with GHD have a gradual worsening in cardiovascular dysfunction [11]. It is therefore not illogical to introduce the second trimester parameters into a prediction model. Women at risk could be considered for preventive treatment such as low-dose aspirin depending on the grade of cardiovascular maladaptation and the predicted first trimester risk profile [28]. But adding a second trimester assessment could confirm more accurately the risk status and determine a personalized follow up monitoring, such as remote monitoring [29-31] and/or tailored hemodynamic-based antihypertensive therapy [32].

Gestational hypertensive disorders are not only linked to cardiovascular maladaptations throughout a pregnancy, but predisposes also to cardiovascular diseases in later life, including a potential hypertensive risk in a future pregnancy [33, 34]. Understanding completely the behaviour of the cardiovascular system before, during and after a pregnancy together with the knowledge of correct measuring these biophysical parameters, could contribute to the advancement of the current approach of gestational hypertensive disorders. It is important to screen patients in time to possibly prevent or at least delay the hypertensive problem.

These first preliminary results of our approach look very promising, but further research is absolutely necessary to validate the real performance. It offers a possible new way of solving the limitations in the currently investigated prediction models based on biochemical parameters. Reported incidence of hypertensive disorders of pregnancy varies between countries, ranging between $1.4-4.0 \%$ for preeclampsia overall, $0.3-0.7 \%$ for early onset preeclampsia and $3.6-9.1 \%$ for gestational hypertension [35]. Incidences are very similar in our study population, with $3.8 \%$ (37/969) for preeclampsia overall, $0.8 \% \quad(8 / 969)$ for early onset preeclampsia and 3.6\% (35/969) for gestational hypertension respectively. As such, our study population could be considered representative for a general obstetric population, however it is to be recognized that we excluded pregnancies complicated with EH, superimposed late preeclampsia with or without SGA, HELLP with or without SGA, isolated SGA and multiplets from the prediction model analysis. Next to this, the Achilles' heel of our screening method is maternal venous Doppler sonography, which not only is subject to human error but also vulnerable for a variety of interfering anatomo-physiologic factors such as respiration, muscle tension, anatomical variability, etc.... [36]. High inter- and intra-observer variability is reported for maternal venous Doppler measurements, markedly improving to intraclass correlations $>0.9$ with the use of (a) an ECG signal as a reference for Doppler wave interpretation, (b) repeated measures and (c) sonographer's training [17]. As such, the performance of our screening algorithm in a general population is to be explored independently in a new prospective assessment, with consideration of sonographer's training, performance and availability, of patient's access and costs, of audit and feedback mechanisms and of impact on overall population health and public costs [37, 38]. The presented prediction models are therefore certainly not ready yet for use in clinical practice in its current form. This paper inspires hopefully other clinicians and researchers to investigate the first and/or second trimester maternal hemodynamics and its prediction potential.

\section{Conclusions}

This is the first study that uses the cardiovascular physiology as fundament for a screening model for hypertensive disorders. It includes only biophysical and demographic parameters, showing prudently good performances (AUC > 94\%). This study is very topical, because there is an urgent need for a good performing screening model to identify not only the preeclamptic population, but any patient who could suffer from hypertension. Experiencing hypertension in a pregnancy is a forerunner of a bad cardiovascular health in later life. Preventing or delaying it is of utmost importance. This study might bring a potential new and fresh input in the current research for screening models. 


\section{Appendix}

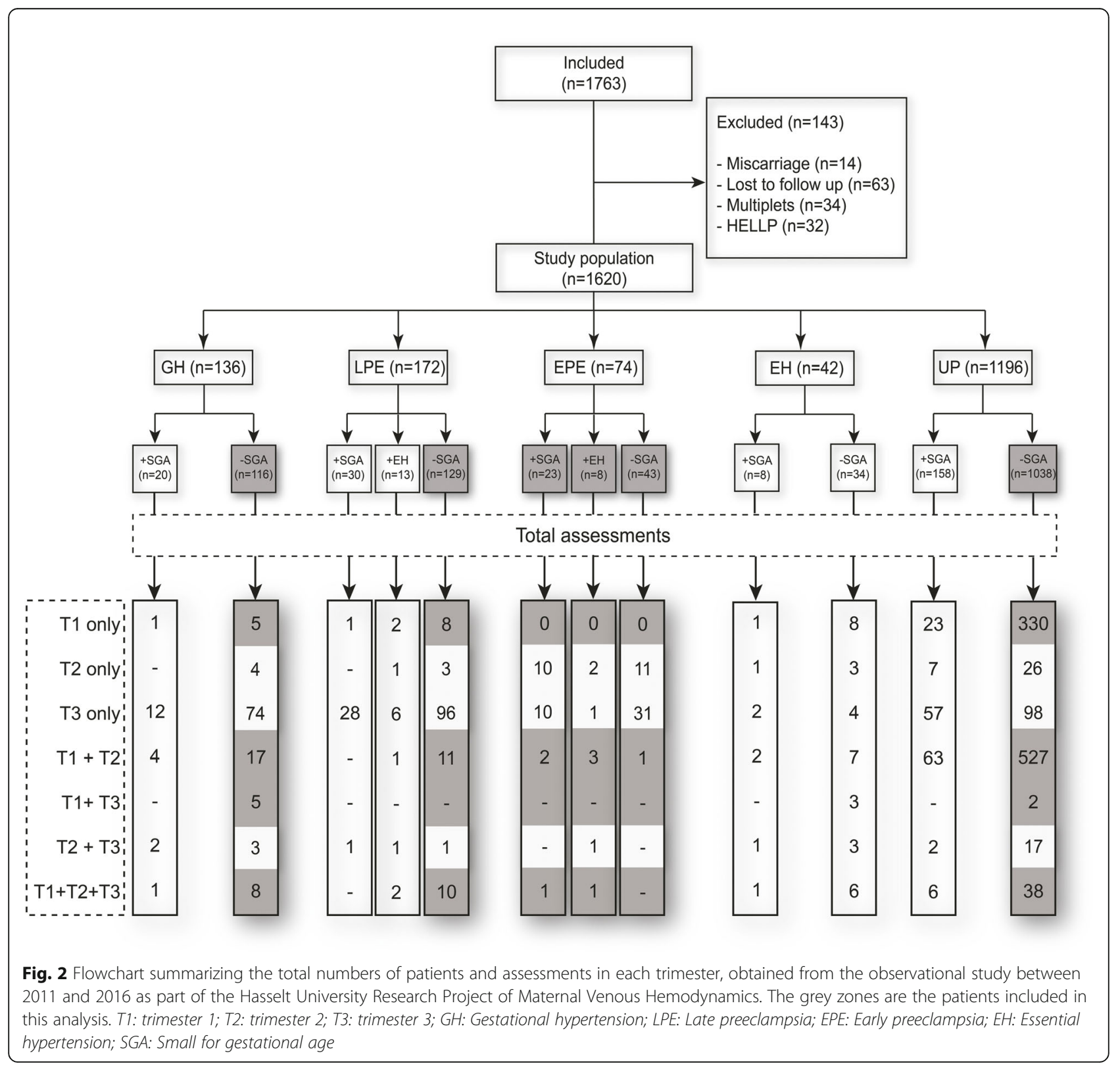

\section{Abbreviations}

ACl: Acceleration index; APT: Arterial pulse transit; AUC: Area under the curve; BMI: Body mass index; CO: Cardiac output; DBP: Diastolic Blood Pressure; DR: Detection ratio; ECW: Extracellular water; EH: Essential hypertension; EPE: Early preeclampsia; FPR: False positive ratio; GH: Gestational hypertension; GHD: Gestational hypertensive disorders; HR: Heart rate; HVI: Hepatic vein index; ICW: Intracellular water; LPE: Late preeclampsia; LR: Likelihood ratios; LVET: Left Ventricular Ejection Time; MAP: Mean arterial pressure; MoM: Multiples of expected normal median; NICCOMO: Non-Invasive Continuous Cardiac Output Monitor;

NP: Normotensive pregnancy; NPV: Negative predictive value; PAPP-

A: Pregnancy-associated plasma protein A; PEP: Pre-ejection period; PI: Pulsatility index; PIGF: placental growth factor; PLS-DA: Partial Least Square Discriminant Analysis; PPV: Positive predictive value; RI: Resistivity
Index; RIVI: Renal interlobar vein index; ROC: Receiver operating curves; sFlt-1: Soluble FMS-like tyrosine kinase-1; SGA: Small for gestational age; SV: Stroke volume; TBW: Total Body Water; TPR: Total peripheral resistance; UFPPR: Uterine flow promoting peripheral resistance; VI: Velocity index; VPT: Venous pulse transit

\section{Acknowledgments}

All authors are part of the Limburg Clinical Research Project (LCRP) at Hasselt University, Belgium.

\section{Funding}

The first author of this work is funded by a Ph.D. grant of the Agency for Innovation by Science and Technology (IWT) in Brussels, Belgium. 


\section{Availability of data and materials}

The datasets used and/or analysed during the current study are available from the corresponding author on reasonable request.

\section{Authors' contributions}

WG + SV: study design, patient inclusion, data management, writing the article. LB: statistics. ASS, DL, KT, JO: patient inclusion. All authors read and approved the final manuscript.

\section{Ethics approval and consent to participate}

Subjects have given their written informed consent and the study protocol was approved by the local ethical committee (MEC ZOL, reference: 13/090 U).

\section{Consent for publication}

Not applicable.

\section{Competing interests}

The authors declare that they have no competing interests.

\section{Publisher's Note}

Springer Nature remains neutral with regard to jurisdictional claims in published maps and institutional affiliations.

\begin{abstract}
Author details
${ }^{1}$ Faculty of Medicine and Life Sciences, Hasselt University, Agoralaan, 3590 Diepenbeek, Belgium. ${ }^{2}$ Department of Obstetrics \& Gynaecology, Ziekenhuis Oost-Limburg, Schiepse Bos 6, 3600 Genk, Belgium. 'Department Physiology, Hasselt University, Agoralaan, 3590 Diepenbeek, Belgium. ${ }^{4}$ Interuniversity Institute for Biostatistics and statistical Bioinformatics, Hasselt University, Agoralaan, 3590 Diepenbeek, Belgium.
\end{abstract}

Received: 30 September 2018 Accepted: 5 May 2019

\section{Published online: 20 May 2019}

\section{References}

1. Visentin S, Londero AP, Camerin M, Grisan E, Cosmi E. A possible new approach in the prediction of late gestational hypertension: the role of the fetal aortic intima-media thickness. Medicine (Baltimore). 2017;96(2):e5515.

2. Tranquilli AL, Dekker G, Magee L, Roberts J, Sibai BM, Steyn W, et al. The classification, diagnosis and management of the hypertensive disorders of pregnancy: a revised statement from the ISSHP. Pregnancy Hypertens. 2014;4(2):97-104

3. O'Gorman N, Wright D, Poon LC, Rolnik DL, Syngelaki A, de Alvarado M, et al. Multicenter screening for pre-eclampsia by maternal factors and biomarkers at 11-13 weeks' gestation: comparison with NICE guidelines and ACOG recommendations. Ultrasound Obstet Gynecol. 2017;49(6):756-60

4. Crovetto F, Figueras F, Triunfo S, Crispi F, Rodriguez-Sureda V, Dominguez C, et al. First trimester screening for early and late preeclampsia based on maternal characteristics, biophysical parameters, and angiogenic factors. Prenat Diagn. 2015;35(2):183-91.

5. Poon LC, Kametas NA, Maiz N, Akolekar R, Nicolaides KH. First-trimester prediction of hypertensive disorders in pregnancy. Hypertension (Dallas, Tex . 1979). 2009;53(5):812-8

6. Giguere Y, Masse J, Theriault S, Bujold E, Lafond J, Rousseau F, et al. Screening for pre-eclampsia early in pregnancy: performance of a multivariable model combining clinical characteristics and biochemical markers. BJOG. 2015;122(3):402-10.

7. Bahado-Singh RO, Syngelaki A, Akolekar R, Mandal R, Bjondahl TC, Han B, et al. Validation of metabolomic models for prediction of early-onset preeclampsia. Am J Obstet Gynecol. 2015;213(4):530 e1-e10.

8. Brandao AH, Evangelista AA, Martins RM, Leite HV, Cabral AC. Prediction of early and late preeclampsia by flow-mediated dilation of the brachial artery. Radiol Bras. 2014:47(4):206-9.

9. Erez O, Romero R, Maymon E, Chaemsaithong P, Done B, Pacora P, et al. The prediction of late-onset preeclampsia: results from a longitudinal proteomics study. PLoS One. 2017;12(7):e0181468.

10. Vonck S, Staelens AS, Bollen I, Broekx L, Gyselaers W. Why non-invasive maternal hemodynamics assessment is clinically relevant in early pregnancy: a literature review. BMC Pregnancy Childbirth. 2016;16(1):302.

11. Gyselaers W, Vonck S, Staelens AS, Lanssens D, Tomsin K, Oben J, et al. Gestational hypertensive disorders show unique patterns of circulatory deterioration with ongoing pregnancy. Am J Physiol Regul Integr Comp Physiol. 2019;316(3):R210-r21.

12. Tomsin K, Mesens T, Molenberghs G, Gyselaers W. Impedance cardiography in uncomplicated pregnancy and pre-eclampsia: a reliability study. J Obstet Gynaecol. 2012;32(7):630-4.

13. Gyselaers W, Molenberghs G, Van Mieghem W, Ombelet W. Doppler measurement of renal interlobar vein impedance index in uncomplicated and preeclamptic pregnancies. Hypertens Pregnancy. 2009;28(1):23-33.

14. Gyselaers W, Molenberghs G, Mesens T, Peeters L. Maternal hepatic vein Doppler velocimetry during uncomplicated pregnancy and pre-eclampsia. Ultrasound Med Biol. 2009:35(8):1278-83.

15. Klabunde R. Vascular function cardiovascular physiology concepts. 5. 2nd ed. Baltimore: Lippincott Williams \& Wilkins; 2012. p. 97.

16. Medis. Impedance Cardiography with ACM Technology. IImenau, Germany [Available from: https://medis.company/cms/uploads/PDF/niccomo.pdf.

17. Staelens AS, Tomsin K, Oben J, Mesens T, Grieten L, Gyselaers W. Improving the reliability of venous Doppler flow measurements: relevance of combined ECG, training and repeated measures. Ultrasound Med Biol. 2014;40(7):1722-8.

18. Tomsin K, Mesens T, Molenberghs G, Gyselaers W. Venous pulse transit time in normal pregnancy and preeclampsia. Reprod Sci (Thousand Oaks, Calif). 2012:19(4):431-6.

19. Vonck S, Staelens AS, Mesens T, Tomsin K, Gyselaers W. Hepatic hemodynamics and fetal growth: a relationship of interest for further research. PLoS One. 2014;9(12):e115594.

20. Nelson TR, Pretorius DH. The Doppler signal: where does it come from and what does it mean? AJR Am J Roentgenol. 1988;151(3):439-47.

21. Gyselaers W, Staelens A, Mesens T, Tomsin K, Oben J, Vonck S, et al. Maternal venous Doppler characteristics are abnormal in pre-eclampsia but not in gestational hypertension. Ultrasound Obstet Gynecol. 2015;45(4):421-6.

22. Staelens AS, Vonck S, Molenberghs G, Malbrain ML, Gyselaers W. Maternal body fluid composition in uncomplicated pregnancies and preeclampsia: a bioelectrical impedance analysis. Eur J Obstet Gynecol Reprod Biol. 2016;204:69-73.

23. Staelens A, Tomsin K, Grieten L, Oben J, Mesens T, Spaanderman M, et al. Non-invasive assessment of gestational hemodynamics: benefits and limitations of impedance cardiography versus other techniques. Expert Rev Med Devices. 2013;10(6):765-79.

24. Yasuda R, Takeuchi K, Funakoshi T, Maruo T. Bioelectrical impedance analysis in the clinical management of preeclamptic women with edema. J Perinat Med. 2003;31(4):275-80.

25. Vinayagam D, Bowe $S$, Sheehan E, Thilaganathan B, Khalil A. Non-invasive Haemodynamic monitoring in pregnancy: a comparative study using ultrasound and bioreactance. Fetal Diagn Ther. 2017;41(4):273-82.

26. Bulletins--Obstetrics ACOP. ACOG practice bulletin. Diagnosis and management of preeclampsia and eclampsia. Number 33, January 2002 Obstet Gynecol. 2002;99(1):159-67.

27. Organization. WH. WHO recommendations for prevention and treatment of pre-eclampsia and eclampsia. 2011.

28. Poon LC, Wright D, Rolnik DL, Syngelaki A, Delgado JL, Tsokaki T, et al. Aspirin for evidence-based preeclampsia prevention trial: effect of aspirin in prevention of preterm preeclampsia in subgroups of women according to their characteristics and medical and obstetrical history. Am J Obstet Gynecol. 2017.

29. Lanssens D, Vandenberk T, Smeets CJ, De Canniere H, Molenberghs G, Van Moerbeke $A$, et al. Remote monitoring of hypertension diseases in pregnancy: a pilot study. JMIR Mhealth Uhealth. 2017;5(3):e25.

30. Lanssens D, Vonck S, Storms V, Thijs IM, Grieten L, Gyselaers W. Corrigendum to "the impact of a remote monitoring program on the prenatal follow-up of women with gestational hypertensive disorders". Eur J Obstet Gynecol Reprod Biol. 2018;223:72-8 European journal of obstetrics, gynecology, and reproductive biology. 2018;231:286-7.

31. Lanssens D, Vonck S, Vandenberk T, Schraepen C, Storms V, Thijs IM, et al. A prenatal remote monitoring program in pregnancies complicated with gestational hypertensive disorders: what are the contributors to the cost savings? Telemed J E Health. 2018.

32. Lees C, Ferrazzi E. Relevance of Haemodynamics in treating pre-eclampsia. Curr Hypertens Rep. 2017;19(9):76.

33. Carty DM, Delles C, Dominiczak AF. Preeclampsia and future maternal health. J Hypertens. 2010;28(7):1349-55.

34. Ghossein-Doha C, Spaanderman M, van Kuijk SM, Kroon AA, Delhaas T, Peeters $L$. Long-term risk to develop hypertension in women with former 
preeclampsia: a longitudinal pilot study. Reprod Sci (Thousand Oaks, Calif). 2014;21(7):846-53.

35. Roberts CL, Ford JB, Algert CS, Antonsen S, Chalmers J, Cnattingius S, et al. Population-based trends in pregnancy hypertension and pre-eclampsia: an international comparative study. BMJ Open. 2011;1(1):e000101.

36. Gyselaers W. Maternal venous hemodynamic dysfunction in Proteinuric gestational hypertension: evidence and implications. J Clin Med. 2019;8(3):335.

37. Gyselaers WJ, Vereecken AJ, Van Herck EJ, Straetmans DP, de Jonge ET,

Ombelet WU, et al. Population screening for fetal trisomy 21: easy access to screening should be balanced against a uniform ultrasound protocol. Prenat Diagn. 2005;25(11):984-90

38. Neyt M, Hulstaert F, Gyselaers W. Introducing the non-invasive prenatal test for trisomy 21 in Belgium: a cost-consequences analysis. BMJ Open. 2014;4(11):e005922.

Ready to submit your research? Choose BMC and benefit from:

- fast, convenient online submission

- thorough peer review by experienced researchers in your field

- rapid publication on acceptance

- support for research data, including large and complex data types

- gold Open Access which fosters wider collaboration and increased citations

- maximum visibility for your research: over $100 \mathrm{M}$ website views per year

At BMC, research is always in progress.

Learn more biomedcentral.com/submissions 